\title{
On the Riccati transfer matrix method for repetitive structures
}

\author{
N.G. Stephen ${ }^{1}$ \\ School of Engineering Sciences, Mechanical Engineering, University of Southampton, \\ Highfield, Southampton SO17 1BJ, UK
}

\begin{abstract}
The Riccati transfer matrix method is employed in the elastostatic analysis of a repetitive structure subject to various loadings; the eigenvalues of particular terms featuring in the recursive relationships show why the method is numerically stable.
\end{abstract}

Keywords: Riccati; transfer matrix; repetitive structure.

\section{Introduction}

A structure is said to be repetitive, or periodic, when its construction takes the form of a spatially repeated cell. Since the manufacture and construction, or assembly, of such structures is also a repetitive process, they often represent a cost effective design solution in many mechanical, aerospace and civil engineering applications. Eigenanalysis of the transfer matrix $\mathbf{G}$ of the single repeating cell provides an efficient means of characterising and analysing its elastostatic behaviour. Non-unity eigenvalues are the rates of decay of selfequilibrated loading, as anticipated by Saint-Venant's principle, and occur as reciprocals according to whether decay is from left-to-right, or vice-versa. Multiple unity eigenvalues pertain to the transmission of load, e.g. tension, or bending moment, as well as the rigid-body displacements and rotations; equivalent continuum properties, such as cross-sectional area, second moment of area and Poisson's ratio, can be determined from the associated eigen- and principal vectors. However, when a complete structure is to be analysed, one typically has a two-point boundary value problem (TPBVP). Thus for a tip-loaded cantilever, the load vector at the tip, $\mathbf{F}(N)$, and the displacement vector at the fixed root, $\mathbf{d}(0)$, will be known; the displacement vector at the tip, $\mathbf{d}(N)$, and the reaction force vector at the root, $\mathbf{F}(0)$, are unknowns. Such problems are typically ill-conditioned. In a recent paper (Stephen, 2009) the analysis of a cantilevered ten-cell repetitive pin-jointed structure subjected to tip-loading, and to distributed loading with an intermediate support, was described; the need to construct

1 Tel.: +44 (0)23 80592359; fax: +44 (0)23 80593230. E-mail address: ngs@ soton.ac.uk (N.G. Stephen) 
powers of the transfer matrix, up to $\mathbf{G}^{10}$, was seen to be the source of the ill-conditioning, and not the inversion of one partition of the stiffness matrix, as some authors have claimed. This becomes abundantly clear when the cross-sectional state-vector of displacement and force components is expressed in terms of the participation coefficients of the eigen- and principal vectors, and spatial evolution is expressed in terms of powers of the Jordan canonical form. In particular, the eigenvalues of $\mathbf{G}$ are unity with a multiplicity of six, $0.059596,-0.070207$ and 0.28292 , together with their reciprocals $16.780,-14.244$ and 3.5346 , respectively; in the calculation of $\mathbf{G}^{10}$, the term $16.780^{10} \approx 1.8 \times 10^{12}$ then becomes dominant and will magnify any errors. For a fifty-cell repetitive structure, a term $16.780^{50} \approx 1.7 \times 10^{61}$ will render the method wholly inaccurate. Metaphorically, while Saint-Venant's principle (SVP) is physically applicable (self-equilibrating forces decay piecewise exponentially), it is not numerically applicable (numerical errors both decay and increase piecewise exponentially obviously the latter prevails), at least in that formulation.

In the present paper, which should be read in conjunction with Stephen (2009), the Riccati transformation is employed to produce a numerically reliable formulation. Moreover, the eigenvalues of particular terms in the recursive relationships reveal why the method is numerically stable - these are three unity eigenvalues, while the non-unity eigenvalues converge onto $0.059596,-0.070207$ and 0.28292 , which are precisely those of Saint-Venant decay; again metaphorically, SVP is now both physically and numerically applicable.

Horner and Pilkey (1978) provided one of the earliest applications of the Ricatti transformation within structural mechanics, but its use is by no means widespread, despite its evident numerical stability. Xue $(2003,2004)$ employed the transform in terms of a stiffness equation transfer method in elastodynamic problems, while Stephen and Wang (2000), who were at that time unaware of the approach, attempted various force and displacement transfer matrix formulations for elastostatic problems related to the present structure. Numerical issues associated with elastodynamic transfer matrix analysis of waveguides have recently been reported by Waki et al (2009). On the other hand, the so-called algebraic Riccati equation is familiar to the control engineering community in relation to optimal control; the TPBVP then consists, typically, of a known initial state-vector $\mathbf{x}(0)$, and a control law $\mathbf{u}(t)$ is sought to achieve some required final state $\mathbf{x}(T)$ over some period of time $T$, which may be finite or infinite, subject to the minimisation of some cost-functional, $J$. The TPBVP is replaced by two one-point boundary value problems, one solved backwards in time from the 
final state $\mathbf{x}(T)$, the second forward in time from the initial state $\mathbf{x}(0)$. For the present structural mechanics example, this process is spatial rather than temporal.

\section{Theory}

The stiffness matrix $\mathbf{K}$ of the single cell relates the force and displacement vectors on either side as $\mathbf{F}=\mathbf{K} \mathbf{d}$, or in partitioned form

$$
\left[\begin{array}{l}
\mathbf{F}_{\mathrm{L}} \\
\mathbf{F}_{\mathrm{R}}
\end{array}\right]=\left[\begin{array}{ll}
\mathbf{K}_{\mathrm{LL}} & \mathbf{K}_{\mathrm{LR}} \\
\mathbf{K}_{\mathrm{RL}} & \mathbf{K}_{\mathrm{RR}}
\end{array}\right]\left[\begin{array}{l}
\mathbf{d}_{\mathrm{L}} \\
\mathbf{d}_{\mathrm{R}}
\end{array}\right]
$$

where the subscripts $\mathrm{L}$ and $\mathrm{R}$ denote left and right, respectively. The transfer matrix $\mathbf{G}$ is determined from the stiffness matrix $\mathbf{K}$ according to

$$
\left[\begin{array}{l}
\mathbf{d}_{\mathrm{R}} \\
\mathbf{F}_{\mathrm{R}}
\end{array}\right]=\left[\begin{array}{cc}
-\mathbf{K}_{\mathrm{LR}}^{-1} \mathbf{K}_{\mathrm{LL}} & -\mathbf{K}_{\mathrm{LR}}^{-1} \\
\mathbf{K}_{\mathrm{RL}}-\mathbf{K}_{\mathrm{RR}} \mathbf{K}_{\mathrm{LR}}^{-1} \mathbf{K}_{\mathrm{LL}} & -\mathbf{K}_{\mathrm{RR}} \mathbf{K}_{\mathrm{LR}}^{-1}
\end{array}\right]\left[\begin{array}{c}
\mathbf{d}_{\mathrm{L}} \\
-\mathbf{F}_{\mathrm{L}}
\end{array}\right]=\left[\begin{array}{ll}
\mathbf{G}_{\mathrm{dd}} & \mathbf{G}_{\mathrm{dF}} \\
\mathbf{G}_{\mathrm{Fd}} & \mathbf{G}_{\mathrm{FF}}
\end{array}\right]\left[\begin{array}{c}
\mathbf{d}_{\mathrm{L}} \\
-\mathbf{F}_{\mathrm{L}}
\end{array}\right],
$$

or more compactly $\mathbf{s}_{\mathrm{R}}=\mathbf{G} \mathbf{s}_{\mathrm{L}}$; the force sign convention of finite element analysis (FEA) is employed, hence the negative force vector on the right-hand side of Eq. (2). The transfer matrix thus describes how a state-vector evolves as one moves from the left-hand side of the cell to the right-hand side. For an extended structure, it is more convenient to write this as $\mathbf{s}(n+1)=\mathbf{G} \mathbf{s}(n)$, which relates the state-vector on the right-hand side of the $(n+1)$ th cell to the state-vector on the right-hand side of the $n$th cell. When an external force vector $\mathbf{F}_{\text {ext }}(n)$ is applied at the $n$th nodal cross-section, Eq. (2) becomes (Stephen, 2009)

$$
\left[\begin{array}{c}
\mathbf{d}(n+1) \\
\mathbf{F}(n+1)
\end{array}\right]=\left[\begin{array}{ll}
\mathbf{G}_{\mathrm{dd}} & \mathbf{G}_{\mathrm{dF}} \\
\mathbf{G}_{\mathrm{Fd}} & \mathbf{G}_{\mathrm{FF}}
\end{array}\right]\left[\begin{array}{l}
\mathbf{d}(n) \\
\mathbf{F}(n)
\end{array}\right]-\left[\begin{array}{ll}
\mathbf{G}_{\mathrm{dd}} & \mathbf{G}_{\mathrm{dF}} \\
\mathbf{G}_{\mathrm{Fd}} & \mathbf{G}_{\mathrm{FF}}
\end{array}\right]\left[\begin{array}{c}
\mathbf{0} \\
\mathbf{F}_{\mathrm{ext}}(n)
\end{array}\right] .
$$

Now introduce the Riccati transform: write

$$
\mathbf{F}(n+1)=\mathbf{R}(n+1) \mathbf{d}(n+1)+\mathbf{g}(n+1), \text { and } \mathbf{F}(n)=\mathbf{R}(n) \mathbf{d}(n)+\mathbf{g}(n),
$$

where $\mathbf{R}$ is the Riccati matrix, and $\mathbf{g}$ is a column vector of force components. Next substitute (4b) into the first row of Eq. (3) to give

$$
\mathbf{d}(n+1)=\mathbf{G}_{\mathrm{dd}} \mathbf{d}(n)+\mathbf{G}_{\mathrm{dF}}[\mathbf{R}(n) \mathbf{d}(n)+\mathbf{g}(n)]-\mathbf{G}_{\mathrm{dF}} \mathbf{F}_{\mathrm{ext}}(n)
$$

and both (4a) and (4b) into the second row to give

$$
\mathbf{R}(n+1) \mathbf{d}(n+1)+\mathbf{g}(n+1)=\mathbf{G}_{\mathrm{Fd}} \mathbf{d}(n)+\mathbf{G}_{\mathrm{FF}}[\mathbf{R}(n) \mathbf{d}(n)+\mathbf{g}(n)]-\mathbf{G}_{\mathrm{FF}} \mathbf{F}_{\mathrm{ext}}(n) .
$$

Finally, employ Eq. (5) to eliminate $\mathbf{d}(n+1)$ from Eq. (6) to give 


$$
\begin{gathered}
\mathbf{R}(n+1)\left[\mathbf{G}_{\mathrm{dd}} \mathbf{d}(n)+\mathbf{G}_{\mathrm{dF}}[\mathbf{R}(n) \mathbf{d}(n)+\mathbf{g}(n)]-\mathbf{G}_{\mathrm{dF}} \mathbf{F}_{\mathrm{ext}}(n)\right]+\mathbf{g}(n+1)= \\
\mathbf{G}_{\mathrm{Fd}} \mathbf{d}(n)+\mathbf{G}_{\mathrm{FF}}[\mathbf{R}(n) \mathbf{d}(n)+\mathbf{g}(n)]-\mathbf{G}_{\mathrm{FF}} \mathbf{F}_{\mathrm{ext}}(n) .
\end{gathered}
$$

For this to be true for arbitrary $\mathbf{d}(n)$, one requires both

$$
\mathbf{R}(n+1) \mathbf{G}_{\mathrm{dF}} \mathbf{g}(n)-\mathbf{R}(n+1) \mathbf{G}_{\mathrm{dF}} \mathbf{F}_{\mathrm{ext}}(n)+\mathbf{g}(n+1)=\mathbf{G}_{\mathrm{FF}} \mathbf{g}(n)-\mathbf{G}_{\mathrm{FF}} \mathbf{F}_{\mathrm{ext}}(n)
$$

and

$$
\mathbf{R}(n+1) \mathbf{G}_{\mathrm{dd}}+\mathbf{R}(n+1) \mathbf{G}_{\mathrm{dF}} \mathbf{R}(n)=\mathbf{G}_{\mathrm{Fd}}+\mathbf{G}_{\mathrm{FF}} \mathbf{R}(n),
$$

and hence the recursive relationships

$$
\mathbf{g}(n)=\left[\mathbf{G}_{\mathrm{FF}}-\mathbf{R}(n+1) \mathbf{G}_{\mathrm{dF}}\right]^{-1} \mathbf{g}(n+1)+\mathbf{F}_{\mathrm{ext}}(n)
$$

and

$$
\mathbf{R}(n)=\left[\mathbf{G}_{\mathrm{FF}}-\mathbf{R}(n+1) \mathbf{G}_{\mathrm{dF}}\right]^{-1}\left[\mathbf{R}(n+1) \mathbf{G}_{\mathrm{dd}}-\mathbf{G}_{\mathrm{Fd}}\right]
$$

these two equations lie at the heart of the "backward in space" process. Note that the term $\left[\mathbf{G}_{\mathrm{FF}}-\mathbf{R}(n+1) \mathbf{G}_{\mathrm{dF}}\right]^{-1}$ is common to both expressions and, as will be seen, its eigenvalues reveal why the method is numerically stable.

\section{Examples}

The method is now applied to the two examples considered by Stephen (2009).

\subsection{Ten-cell tip-loaded cantilever}

At the free end, we know that $\mathbf{F}_{\text {ext }}(10)=\left[\begin{array}{lllllll}0 & -1000 & 0 & 0 & 0 & 0\end{array}\right]^{\mathrm{T}}$ and $\mathbf{d}(10) \neq \mathbf{0}$, so set $\mathbf{R}(10)=\mathbf{0}$ in Eq. (4), to give $\mathbf{g}(10)=\mathbf{F}_{\text {ext }}(10)$. The external load vector $\mathbf{F}_{\mathrm{ext}}(n)$ is zero for all other cross-sections, so Eq. (10) yields $\mathbf{g}(9)=\left[\mathbf{G}_{\mathrm{FF}}\right]^{-1} \mathbf{g}(10)$, $\mathbf{g}(8)=\left[\mathbf{G}_{\mathrm{FF}}-\mathbf{R}(9) \mathbf{G}_{\mathrm{dF}}\right]^{-1} \mathbf{g}(9), \mathbf{g}(7)=\left[\mathbf{G}_{\mathrm{FF}}-\mathbf{R}(8) \mathbf{G}_{\mathrm{dF}}\right]^{-1} \mathbf{g}(8)$, etc.

Eq. (11) yields $\mathbf{R}(9)=-\left[\mathbf{G}_{\mathrm{FF}}\right]^{-1} \mathbf{G}_{\mathrm{Fd}}, \mathbf{R}(8)=\left[\mathbf{G}_{\mathrm{FF}}-\mathbf{R}(9) \mathbf{G}_{\mathrm{dF}}\right]^{-1}\left[\mathbf{R}(9) \mathbf{G}_{\mathrm{dd}}-\mathbf{G}_{\mathrm{Fd}}\right]$, $\mathbf{R}(7)=\left[\mathbf{G}_{\mathrm{FF}}-\mathbf{R}(8) \mathbf{G}_{\mathrm{dF}}\right]^{-1}\left[\mathbf{R}(8) \mathbf{G}_{\mathrm{dd}}-\mathbf{G}_{\mathrm{Fd}}\right]$, etc.

The solution process is to calculate recursively to $\mathbf{R}(0)$ and $\mathbf{g}(0)$; since the root of the cantilever is assumed fully fixed, one has $\mathbf{d}(0)=\mathbf{0}$, so from Eq. (4), $\mathbf{F}(0)=\mathbf{g}(0)$; this part of the process is "backward in space". To determine the displacement components at any crosssection, one then employs Eq. (5), which is the "forward in space" process. The eigenvalues 
of the term $\left[\mathbf{G}_{\mathrm{FF}}-\mathbf{R}(n+1) \mathbf{G}_{\mathrm{dF}}\right]^{-1}$ are shown in Table 1 for this ten-cell example (in fact, the number of cells is irrelevant); as one moves backward in space from the cantilever tip, the non-unity eigenvalues quickly converge onto $0.059596,-0.070207$ and 0.28292 , which are precisely those of Saint-Venant decay.

\begin{tabular}{|c|c|}
\hline$n+1$ & eigenvalues \\
\hline 10 & $1,1,1,-0.1397,0.1199,0.5034$ \\
\hline 9 & $1,1,1,-0.0706,0.0588,0.3304$ \\
\hline 8 & $1,1,1,-0.0702,0.0596,0.2873$ \\
\hline 7 & $1,1,1,-0.0702,0.0596,0.2829$ \\
\hline 6 & $\vdots$ \\
\hline$\vdots$ &
\end{tabular}

Table 1. Eigenvalues of $\left[\mathbf{G}_{\mathrm{FF}}-\mathbf{R}(n+1) \mathbf{G}_{\mathrm{dF}}\right]^{-1}$ for ten-cell tip-loaded cantilever.

The forward in space expression for the displacements, Eq. (5), can be written as

$$
\mathbf{d}(n+1)=\left[\mathbf{G}_{\mathrm{dd}}+\mathbf{G}_{\mathrm{dF}} \mathbf{R}(n)\right] \mathbf{d}(n)+\mathbf{G}_{\mathrm{dF}}\left[\mathbf{g}(n)-\mathbf{F}_{\mathrm{ext}}(n)\right]
$$

and the term $\left[\mathbf{G}_{\mathrm{dd}}+\mathbf{G}_{\mathrm{dF}} \mathbf{R}(n)\right]$ has the same eigenvalues as shown in Table 1, thus the numerical process, both backward and forward in space, may be regarded as an analogue of the physical application of SVP.

The method has also been applied to a fifty-cell tip-loaded cantilever, and the displacement components are in perfect agreement with what may be regarded as exact FEA predictions; in contrast, the approach described by Stephen (2009) for a fifty-cell structure was noted to be wholly inaccurate.

\subsection{Distributed loading with intermediate support}

For the same ten-cell structure, one now has a vertical downward force of $1 \mathrm{kN}$ applied at each cross-section, and a simple-support at the 7th nodal cross-section. Thus

$$
\begin{aligned}
& \mathbf{F}_{\text {ext }}(n)=\left[\begin{array}{llllll}
0 & -1000 & 0 & 0 & 0 & 0
\end{array}\right]^{\mathrm{T}} \text { for } n=1,2, \cdots, 6,8,9,10 ; \text { for } n=7 \text {, one has } \\
& \mathbf{F}_{\text {ext }}(7)=\left[\begin{array}{llllll}
0 & -1000 & 0 & 0 & 0 & F
\end{array}\right]^{\mathrm{T}} \text { where } F \text { is the unknown support reaction required to }
\end{aligned}
$$


reduce the vertical displacement of this node to zero. The process again employs Eqs. (10) and (11) to calculate all the $\mathbf{R}$ matrices and $\mathbf{g}(0)$; the latter now contains $F$ as an unknown. Eq. (5) is then employed to calculate displacement vectors up to $\mathbf{d}(7)$, and the relevant component is calculated as $-232527.5+31.8 F$; for this to be zero requires $F=7312.13 \mathrm{~N}$. The remainder of the calculation is then straightforward, and agreement with FE predictions is perfect.

\section{Conclusions}

A recent paper, Stephen (2009), extended the transfer matrix approach to a repetitive (or periodic) structure subject to distributed loading, and with an intermediate support; numerical inaccuracies were clearly seen to be associated with powers of greater than unity eigenvalues of the transfer matrix. In the present note, the same examples have been treated using the Riccati transfer matrix method, and the eigenvalues of particular terms within the recursive relationships show why the method is numerically stable.

\section{References}

Horner GC, Pilkey WD. The Riccati transfer matrix method. ASME J. Mech. Des. 1978; 100(2): 297-302.

Mufti IH, Chow CK, Stock FT. Solution of ill-conditioned linear two-point boundary value problems by the Riccati transformation. SIAM Rev. 1969; 11(4): 616-619.

Stephen NG. Repetitive beam-like structures: Distributed loading and intermediate support. Int. J. Solids Struct. 2009; 46(20): 3664-3668.

Stephen NG, Wang PJ. On transfer matrix eigenanalysis of pin-jointed frameworks. Comput. Struct. 2000; 78(4): 603-615.

Waki Y, Mace BR, Brennan MJ. Numerical issues concerning the wave and finite element method for free and forced vibrations of waveguides. J. Sound Vib. 2009; 327(1-2): 92-109. Xue H. A stiffness equation transfer method for natural frequencies of structures. J. Sound Vib. 2003; 268(5): 881-895.

Xue H. A stiffness equation transfer method for transient dynamic response analysis of structures. J. Sound Vib. 2004; 273(4-5): 1063-1078. 


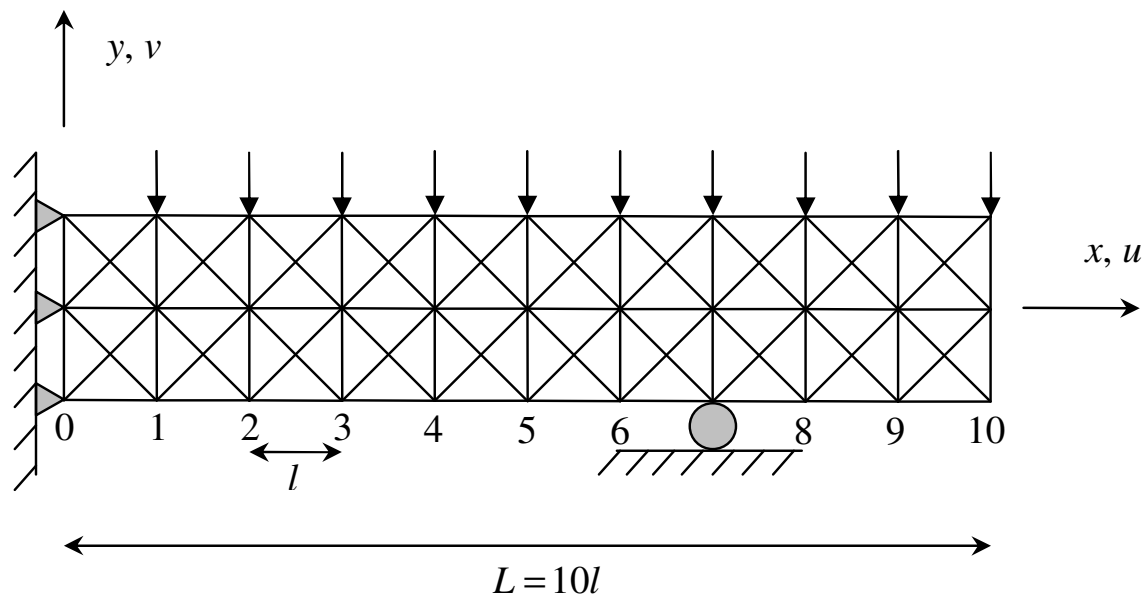

Figure 1. Ten-cell repetitive structure subject to distributed surface loading, with an intermediate support at the 7 th nodal cross-section. Each downward arrow represents a force of $1 \mathrm{kN}$. 